\title{
Assessment of immunization procedures for foot-and-mouth disease in large-scale pig farms in China based on actual data and dynamics
}

\author{
Huarong Ren ${ }^{1,2,3}$, Zhen $\mathrm{Jin}^{1,2}$, Xin $\mathrm{Pei}^{4}$, Mingtao $\mathrm{Li}^{4}$, Youming Wang ${ }^{5}$ and Juan Zhang ${ }^{1,2^{*}}$
}

\begin{abstract}
Foot-and-mouth disease (FMD) is an acute, highly infectious and pathogenic animal disease. In recent years, with the rapid development of the swine breeding industry in China, pig farms have shown a trend of larger-scale development. Large-scale pig farms employ standardized management, a high level of automation, and a strict system. However, these farms have a large trading volume, and increased transmission intensity of FMD is noted inside the farm. At present, the main control measure against FMD is pig vaccination. However, a standard for immunization procedures is not available, and currently adopted immunization procedures have not been effectively and systematically evaluated. Taking a typical large-scale pig farm in China as the research subject and considering the breeding pattern, piggery structure, age structure and immunization procedures, an individual-based state probability model is established to evaluate the effectiveness of the immune procedure. Based on numerical simulation, it is concluded that the optimal immunization program involves primary immunization at 40 days of age and secondary immunization at 80 days of age for commercial pigs. Breeding boars and breeding sows are immunized 4 times a year, and reserve pigs are immunized at 169 and 259 days of age. According to the theoretical analysis, the average control reproduction number of individuals under the optimal immunization procedure in the farm is 0.4927 . In the absence of immunization, the average is 1.7498 , indicating that the epidemic cannot be controlled without immunization procedures.
\end{abstract}

Keywords: Pig farm, Foot-and-mouth disease, Individual-based state probability model, Immunization procedure, Infection probability, Individual-based control reproduction number

\section{Introduction}

Foot-and-mouth disease (FMD) is an acute, febrile and highly contagious disease caused by foot-and-mouth disease virus (FMDV). In addition, FMD is a global animal disease (Belsham et al. 2011; Kardjadj 2017). Greater than 70 types of animals are susceptible to FMD, including

*Correspondence: zhangjuan1020@sxu.edu.cn

${ }^{1}$ Complex Systems Research Center, Shanxi University, 030006 Shanxi, China

${ }^{2}$ Shanxi Key Laboratory of Mathematical Techniques and Big Data Analysis on

Disease Control and Prevention, 030006 Shanxi, China

Full list of author information is available at the end of the article major domestic animals, such as pigs, cattle, sheep and other domestic and wild cloven-hoofed animals (Wernery and Kinne 2012). Outbreaks of FMD can cause great economic losses to a country (Carpenter et al. 2011; Thompson et al. 2002). Since 1958, there have been three serotypes of FMDV in China: O, A and Asia 1 (Bai et al. 2011). Recovery from one serotype does not provide immunity to other serotypes (Rweyemamu et al. 2008). In China, serotype O FMDV has persisted since 2010. From 2010 to 2020, a total of 140 outbreaks of FMD were recorded, including serotype O (99 times), serotype A (34 
times) and unspecified serotype (7 times) (Wang et al. 2021).

There are three modes of FMD transmission among individuals: direct contact between individuals, indirect transmission by media and aerosol transmission in the air (Ringa and Bauch 2014; Alexandersen et al. 2003). Pigs infected with FMD need $3-4$ weeks to clear the virus (Alexandersen et al. 2002). During the infection period, some pigs show clinical symptoms and will be culled. Other pigs recover under the action of vaccines (Barnett et al. 2002). To date, the main prevention and control measures of FMD are immunization, which is performed in accordance with several immunological processes. The immune procedure of the farms determines the immune antibody qualified rate, which further affects the prevalence of FMD.

For FMD, the national purification standard in China is that the positive rate of pathogen monitoring is less than or equal to $0.1 \%$ (Ministry of Agriculture 2016). In addition, the national requirement for FMD immunization is that the qualified rate of immune antibodies should be maintained above $70 \%$ year round (Ministry of Agriculture 2020). The qualified rate of immune antibodies refers to the proportion of successfully immunized pigs. At present, no standard FMD vaccination procedure is available. For swine, boars and sows are generally immunized 3 or 4 times a year; pigs should be immunized at least twice during the piglet-nursery-fattening stage (Liu et al. 2019). The first immunization time is usually between 40 and 70 days of age, and the second immunization time is between 80 and 100 days of age. In the present study, the pathogen positive rate, the qualified rate of immune antibodies and basic reproduction number were used as indicators to evaluate the effectiveness of the immune procedure for serotype O FMDV in pig farms.

The pig breeding modes in China mainly include freerange breeding and large-scale breeding. In recent years, free-range farmers have gradually been eliminated or developed for large-scale breeding. According to the data in China Animal Husbandry and Veterinary Yearbook, the number of farms in 2017 was 37,746,624, which is down $54 \%$ from 2007. In 2014, the proportion of large-scale farms was $41.8 \%$, which increased by $7.2 \%$ compared with 2010 (Ministry of Agriculture 2017). However, due to the higher breeding density of large-scale farms, an infected individual on a large-scale farm has a greater transmission intensity inside the farm. In 2011, the draft statement of Measures for the Supervision and Administration of Transprovincial Transfer of Animals noted that $70 \%$ of animal diseases were related to the movement of animals. Due to the increased frequency of trading on large-scale farms, once an epidemic occurs, the transmission speed and the infection scale will be greater. In this case, it will be more difficult to control the disease, and correspondingly, leading to a higher loss. Therefore, in the present study, we take a typical large-scale farm as the research object to study the transmission and control of FMDV in the farm.

Some works have applied mathematical models to investigate the effects of different immunization strategies (Ringa and Bauch 2014; Wada et al. 2017; Mushayabasa 2020). However, there is no work that applies mathematical models to assess immune procedures in farms. To investigate immune procedures, the individual-based state probability model is the most accurate model and has been widely used in the study of disease transmission dynamics (Gomez et al. 2010; Pei et al. 2019). The individual-based state probability model based on discrete-time Markov chain allows us to obtain the infection and immune status of each pig in the farm.

In the present study, we take a typical large-scale pig farm in China as the research subject. The farm is a leading municipal enterprise that gathers pig breeding, improvement and sale as one. Its annual output of pigs is 60000 heads. The pigs in this farm can be roughly divided into four types: commercial pigs, reserve pigs, breeding sows, and breeding boars. Commercial pigs are categorized into piglets, nursery pigs and fattening pigs according to days of age. Breeding sows are classified as pregnant sows, lactating sows, and nonpregnant sows according to the production process. Reserve pigs are classified as reserve boars and replacement gilts. According to the actual situation, different types of pigs are raised in different houses. Each pig house is divided into several pens, and a fixed number of pigs are placed in each pen. The types of pig houses, the corresponding types of pigs, the corresponding number of pens, and the number of pigs in each pen are shown in Table S1 in Additional file 1 . The amount of the various pigs on hand is presented in Table S2 in Additional file 1. Piglets are $1 \sim 5$ week-old commercial pigs raised in the delivery room. Nursery pigs are 6-12 week-old commercial pigs raised in a nursery house. Fattening pigs are 13-25 week-old commercial pigs raised in a fattening house. Replacement gilts and reserve boars are 25-37 week-old gilts and boars. The farm adopts batch production, and new piglets are produced. Pigs are eliminated or sold once a week, and each batch of pigs is transferred between different pig houses every week, according to age and pregnancy. These details are introduced in Additional file 2. At present, commercial pigs are vaccinated for the first time at 60 days of age and again at 90 days of age. Reserve pigs in the farm were vaccinated at 169 and 259 days of age. Breeding sows and boars are vaccinated 3 times a year. The disinfection period of the whole farm is approximately one month. 
In the present study, the pig breeding mode and immune procedure of a typical large-scale pig farm in China are introduced first. Second, according to the transmission mechanism of FMD and discrete-time Markov chain, we establish an individual-based state probability model to describe the breeding mode and immune procedure of the farm. Then, through numerical simulation of the model, we predicted the immune and infection processes of the farm in one year and studied the immune antibody qualification rate and positive rate of pathogens in various types of pig houses in the farm under different immune procedures. Finally, the individual-based control reproduction numbers for various types of pig houses and the whole farm under different conditions are calculated and compared.

\section{Results}

\section{Prediction of antibody qualified rate and positive rate}

By applying system (1), which is introduced in the Methods section in detail, the immune antibody qualified rate and the level of pathogen positive rate in this farm within one year are predicted. Figure 1 shows the immune antibody qualified rate for the entire farm and various pig houses in one year. As noted in Fig. 1a, from January 1 to May 19 (139 days), the immune antibody qualified rate in the whole farm will be reduced from 0.8916 to 0.62 . From May 20 to December 31, the immune antibody qualified rate of the whole farm will have two periods, and each period is approximately 120 days (4 months). In these two periods, the minimum qualified rate and average qualified rate of immune antibody in the whole farm did not reach the national requirement (70\%), and the time corresponding to the minimum point was the vaccination time of breeding boars and breeding sows (May 20 and September 20). Therefore, it can be inferred that changing the vaccination time of breeding boars and breeding sows can change the immune antibody qualified rate for the whole farm. As seen from Fig. 1b, the immune antibody qualified rate in the fattening house and the reserve house is relatively high, and the fluctuation of the curve

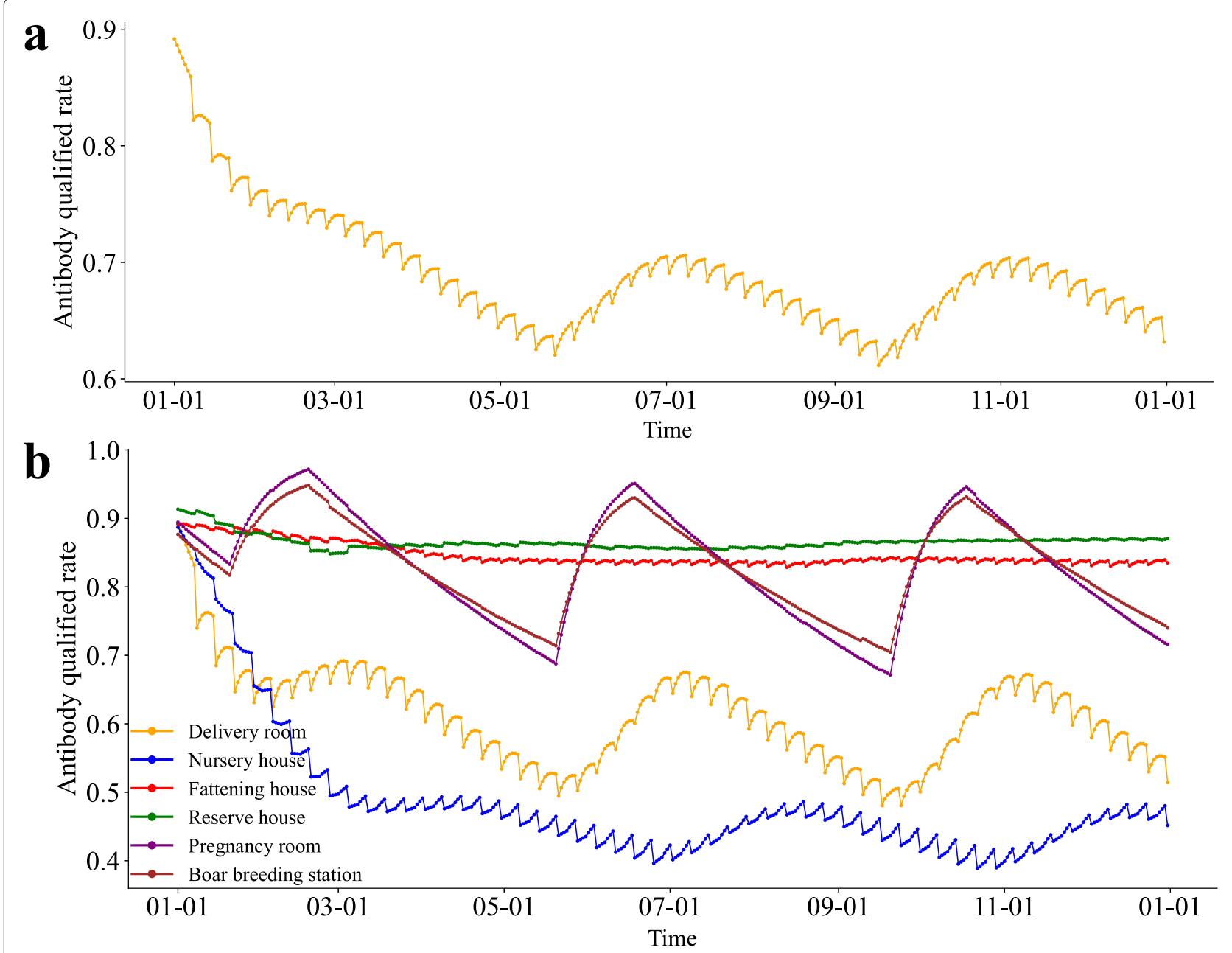

Fig. $1 \mathrm{Imm}$ me antibody qualified rate of the pig farm in one year. a The whole farm. b Different pig houses 
is small. However, the immune antibody qualified rate in the delivery room and the nursery house is relatively low, so it is necessary to increase their immune antibody qualified rate to improve the whole qualified rate. The time of the minimum immune antibody qualified rate in the delivery room is the same as that in the pregnancy house, which is the time of the breeding sows being vaccinated. Therefore, changing the immunization time or frequency of breeding sows can change the immune antibody qualified rate in pregnancy houses and delivery houses. The time difference between the maximum antibody qualified rate in the pregnancy room and the delivery room is approximately 19 days. For breeding boars and sows, the immune antibody qualified rate can reach the maximum at 28 days after vaccination. In addition, given that the breeding rhythm of the farm is 7 days, the immune antibody qualified rate of commercial pigs and reserve pigs are vaccinated in batches and has a small period of 7 days. After May 20, the maximum immune antibody qualified rate in the delivery room and the nursery house does not reach $70 \%$. In addition, the average antibody qualified rate in the pregnancy room is greater than $70 \%$, but the minimum is less than $70 \%$.

As noted in Fig. 2a and b, the positive rate and positive number of the farm will generally show a downward trend in the whole year. The positive rate will be reduced from 0.0083 to $8.83 \times 10^{-5}$. On April 23, the pathogen positive rate will drop to 0.001 . The positive number will be reduced from 125 to 1 . It can be seen from Fig. 2c that the pathogen positive number in all types of pig houses will drop to single digits on March 1. Eventually, the pathogen-positive number in all types of pig houses will be less than 1 . Figure $2 \mathrm{~d}$ shows that the infection probability of pigs in all types of pig houses will increase and then decrease. On January 22, the infection probability of all pig houses will reach the maximum, and its range is [0.00034, 0.00038]. By December 31, the infection probability in all pig houses will be approximately $9.6 \times 10^{-6}$.

\section{Evaluation of immunization procedure}

In this subsection, we examine the effectiveness of immunization programs in the absence of infectious processes. It is assumed that all pigs in the farm at the initial moment are susceptible. Specifically, the probability of any pig being susceptible is 1 , and the probability of being immunized is 0 . Then, we simulate the system (2), which is introduced in the Methods section, to discuss the influence of different primary immunization times, secondary immunization times and immunization frequencies on the immune antibody qualified rate in the farm.

The immune antibody level of breeding sows directly determines the maternal antibody level of piglets. First, we increased the immunization frequency per year for breeding sows and breeding boars to improve the immune antibody qualified rate in the delivery room. When breeding sows and boars are immunized 4 times a year, the corresponding set of immunization times is $T_{v}=$ \{January 20, April 20, July 20, October 20\}. The numerical simulation results of system (2) with different immunization frequencies in one year are shown in Fig. 3. (1) The increase in immunization frequency has no effect on the immune antibody qualified rate in reserve houses and fattening houses (2) and has relatively minimal influence on the immune antibody qualified rate in the nursery house. (3) Obviously, with the increase in immunization frequency, the number of periods of immune antibody qualification in the farm, the delivery room, the pregnancy room and the breeding boar station is increased. (4) In the case of four vaccinations a year, the immune antibody qualified rate in the whole farm and various piggeries shows a periodic steady state after May 20. After May 20, the maximum, minimum and mean immune antibody qualified rates of the whole farm increased by $0.0146,0.0633$ and 0.0273 , respectively. (5) Similarly, the maximum, minimum and mean of the immune antibody qualified rate in the delivery room can increase by $0.0138,0.0984$ and 0.0458 , respectively. Therefore, changing the immunization frequency of breeding sows and breeding boars can change the antibody qualified rate in the delivery room, pregnancy room and breeding boar station and improve the minimum antibody qualified rate in the whole farm. However, this change has a minimal effect on the maximum antibody qualified rate.

When $T_{v}=\{$ January 20, April 20, July 20, October 20 $\}$, the numerical simulation results of system (2) under the different primary immunization times $\left(T_{1} \in\{40,45,50,55,60\}\right)$ of commercial pigs are shown in Fig. 4. Given that piglets in the delivery room are $1-35$ days old, the change in the primary immunization time $T_{1}$ has no effect on the immune antibody qualified rate of piglets in the delivery room. The primary immunization time had no effect on the immune antibody qualified rate in the pregnancy room, reserve house or boar breeding station. Figure 4 shows that the primary immunization time has a greater impact on the antibody qualified rate in the nursery house and the fattening house. As noted in Fig. $4 \mathrm{a}$, when the primary immunization time is earlier, the antibody qualified rate in the nursery house is greater, and the fluctuation range is smaller. When $T_{1}=40$, the antibody qualified rate in the nursery is the largest, the fluctuation is the smallest, and the range at steady state is $[0.583,0.629]$. As noted in Fig. $4 \mathrm{~b}$, the short period of the antibody qualified rate in fattening houses is 7 days. However, the earlier the primary immunization time, the smaller the antibody qualified rate in the 

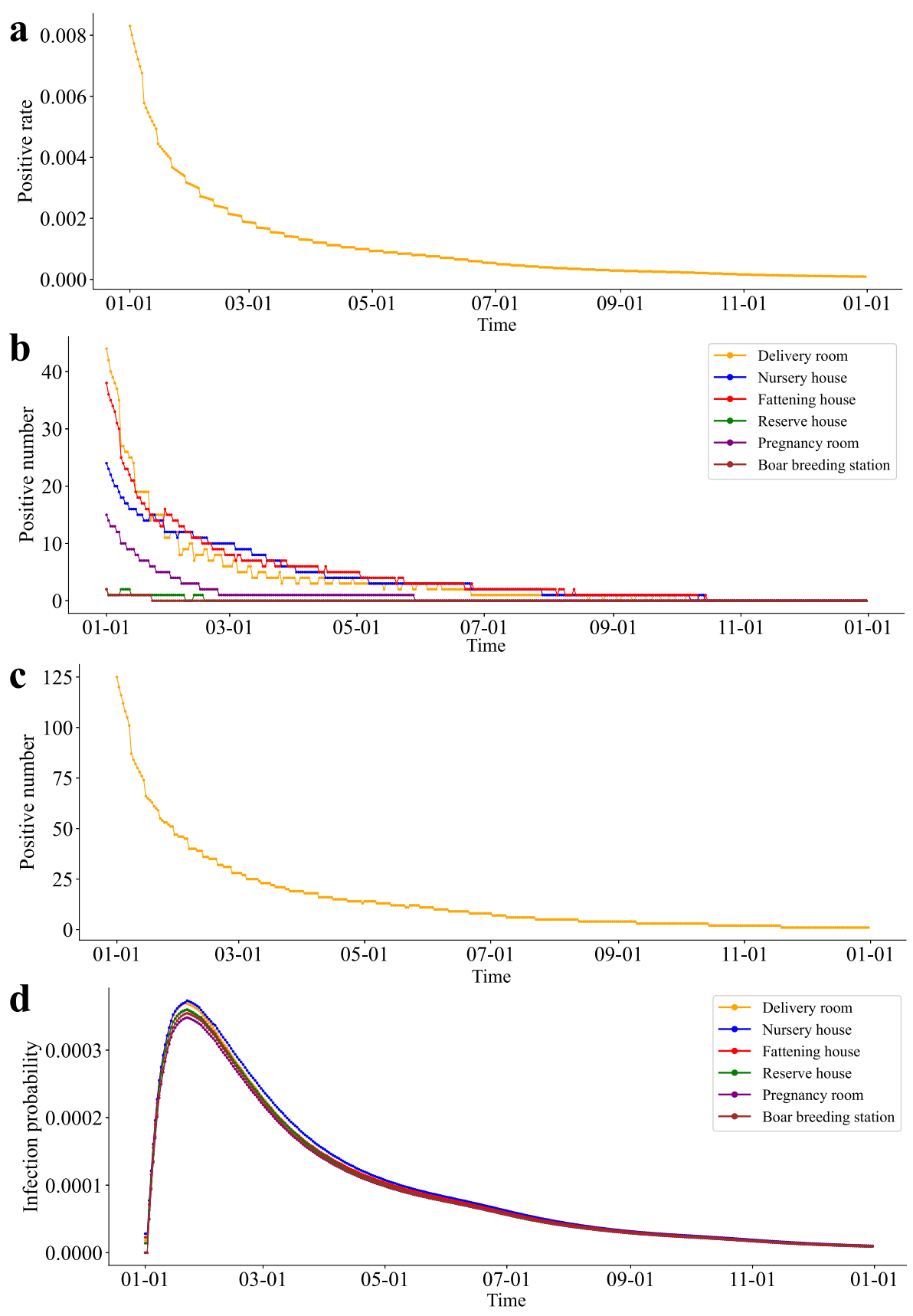

Fig. 2 Infection situation and infection probability of the farm in one year. a The positive rate of the whole farm. $\mathbf{b}$ The positive number of the whole farm. $\mathbf{c}$ The positive number of different kinds of pig houses. $\mathbf{d}$ The infection probability of susceptible pigs in different types of pig houses

fattening house. When $T_{1}=40$, the range of the antibody qualified rate in the fattening house at steady state is $[0.7842,0.8015]$. When $T_{1}=60$, the range at steady state is $[0.8352,0.8458]$. The mean difference of the antibody qualified rate between above two cases is 0.049 .

The immune antibody qualified rate in the fattening house can be altered by changing the secondary immunization time $\left(T_{2}\right)$. When $T_{1}=40$ and $T_{v}=$ \{January 20, April 20, July 20, October 20\}, the numerical simulation results of system (2) under the different secondary immunization times of commercial pigs are shown in Fig. 5. Figure 5 shows the variation of the antibody qualified rate in the whole farm and the fattening house over time. Then, we can obtain the following results: (1) For the whole farm, the earlier the time of secondary immunization, the greater the antibody qualified rate. When $T_{2} \in\{80,85,90,95,100\}$, the minimum antibody qualified rate of the whole farm is less than 


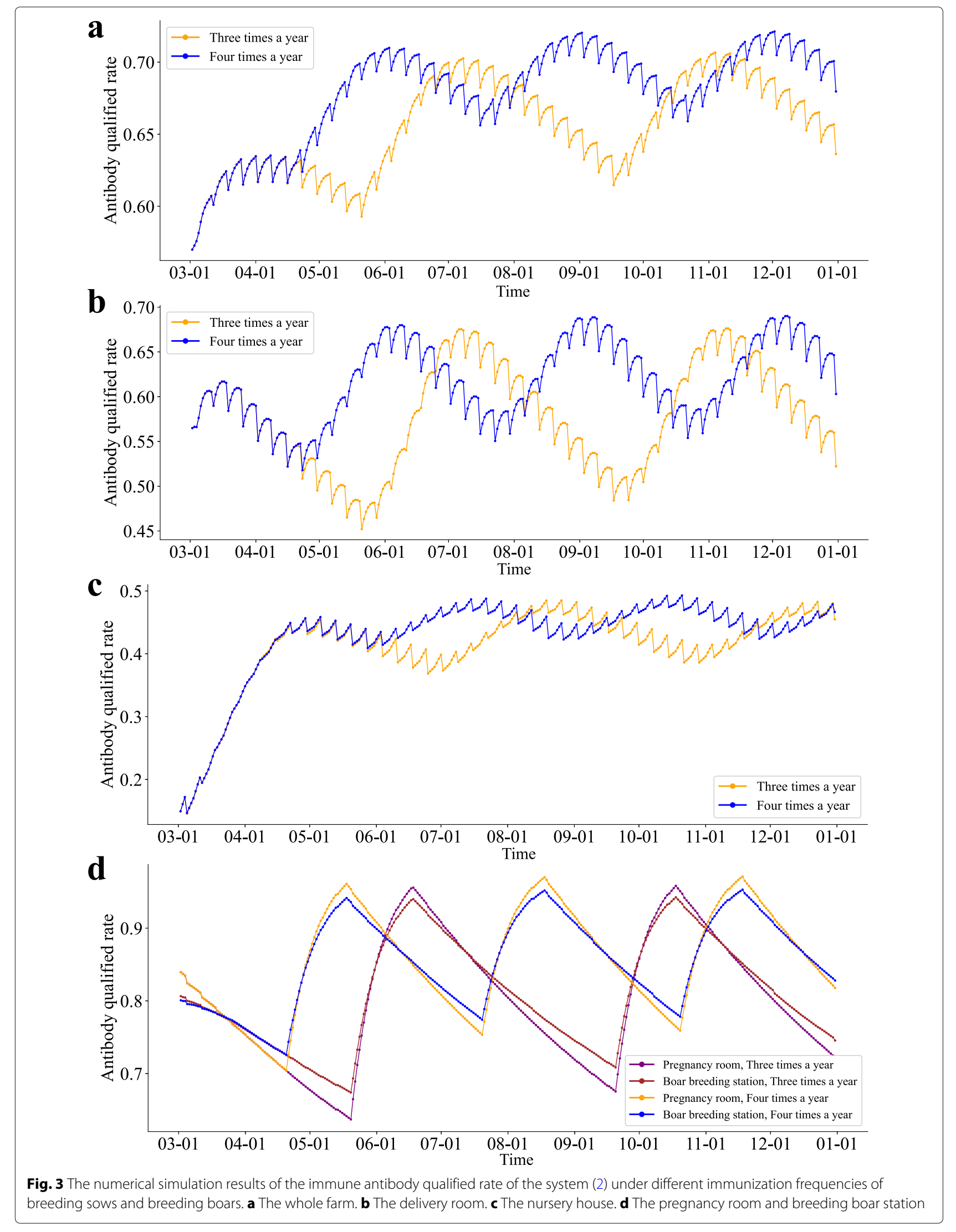




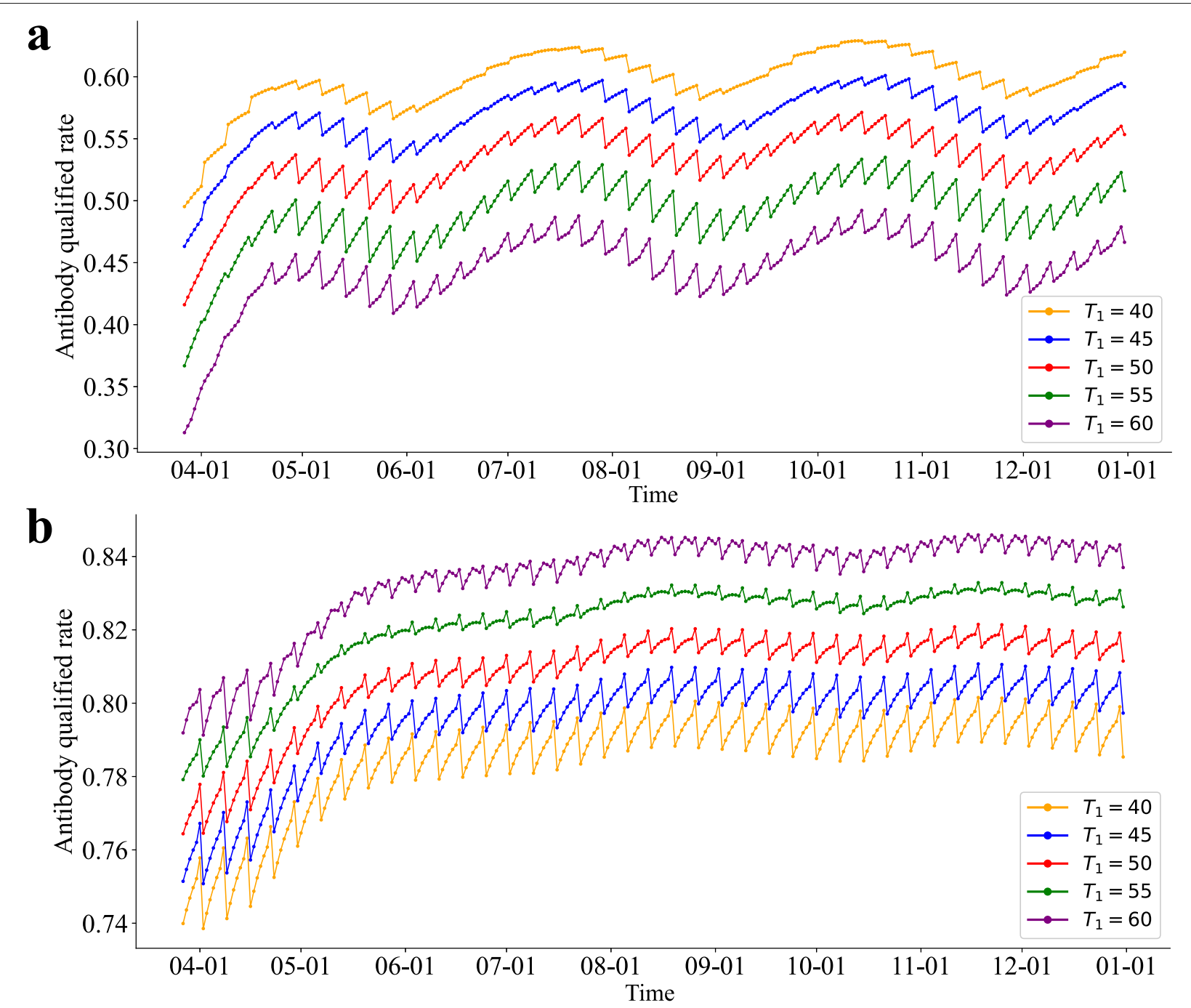

Fig. 4 When $T_{V}=\{$ January 20, April 20, July 20, October 20\}, the numerical simulation results of the antibody qualified rate of the system (2) at different primary immunization times. a The nursery house. $\mathbf{b}$ The fattening house

$70 \%$ after July 20 . However, when $T_{2} \in\{80,85,90,95\}$, the mean antibody qualified rate of the whole farm is greater than $70 \%$ after July 20. (2) The change in the secondary immunization time had no effect on the antibody qualification rate in the delivery room, reserve house, pregnancy room or breeding boar station. (3) Because pigs in the nursery house are $36-84$ days old, when $T_{2} \in$ $\{85,90,95,100\}$, the antibody qualified rate in the nursery house does not change. However, when $T_{2}=80$, the antibody qualified rate increases, its value range after July 20 is $[0.5838,0.6507]$, and its mean value is 0.6157 . (4) The smaller $T_{2}$, the higher the antibody qualified rate in the fattening house. When $T_{2}=80$, the antibody qualified rate is the highest.

According to the above study, a good immune procedure can be obtained: $T_{1}=40, T_{2}=80, T_{v}=$ \{anuary 20, April 20, July 20, October 20\}, $T_{3}=169$ and $T_{4}=259$. With this immune program, considering the spread of FMDV, the system (1) is numerically simulated, and the results are shown in Fig. 6. Compared with the original immune program, the antibody qualified rate in this case increases significantly. The average antibody qualified rate in the whole farm is greater than $70 \%$. The minimum antibody qualified rate in the pregnancy room is greater than $70 \%$. As noted in Fig. 6a and b, the large period of the antibody qualified rate in farms, delivery rooms, pregnancy rooms and boar breeding stations is approximately 91 days. A 21-day difference in the time corresponding to the maximum qualified rate is noted between the delivery room and the pregnancy room. According to Fig. 6c, using this immune produce, the positive rate of the farm will be reduced to less than 


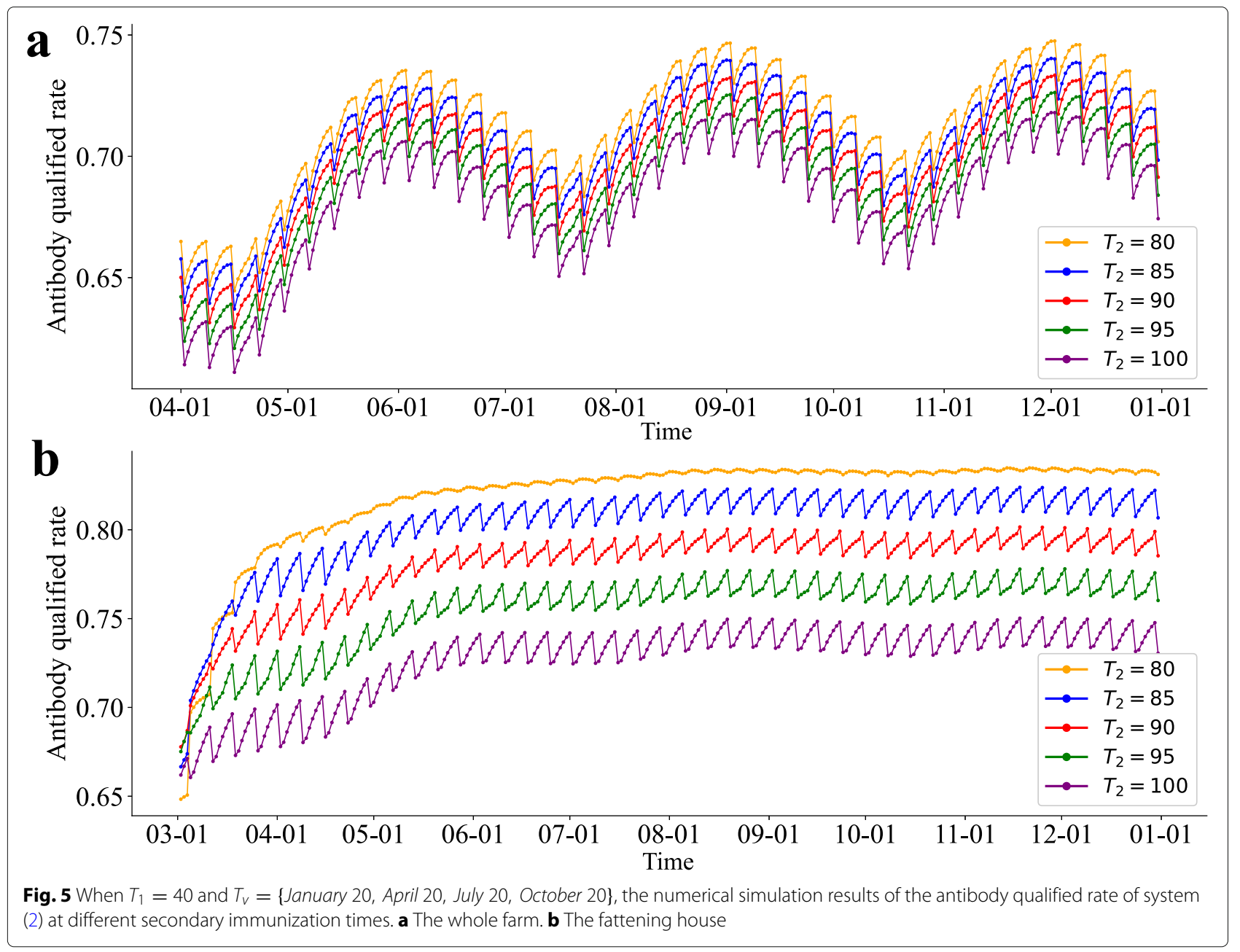

0.001 after April 2 and will be reduced to $2.16 \times 10^{-5}$ by December 31. As noted in Fig. 6d, the infection probability of the susceptible pig in all pig houses first increases and then decreases. On January 21, the infection probability for all pigs will reach the maximum. On December 31, the infection probability for all types of susceptible pigs will be approximately $2.62 \times 10^{-6}$.

\section{Reproduction number}

The reproduction number is divided into the basic reproduction number and the control reproduction number. The basic reproduction number is the number of pigs infected by an infected pig during the average infection period at the beginning of the disease (Dreessche and Watmough 2002). The control reproduction number is defined as the number of pigs infected by an infected pig during the average infection period with immunization measures. The specific calculation formula and its explanation is presented in the Methods section. Now, we consider the individual reproduction number in three cases: no vaccination, immune procedure I, and immune procedure II. The immune procedure I involves the following: Commercial pigs are first immunized at 60 days old. The second immunization is delivered at 90 days old. Breeding boars and breeding sows are immunized thrice a year, and reserve pigs are immunized at 169 and 259 days old. The immune procedure II involves the following: Commercial pigs are first immunized at 40 days old, and the second immunization is delivered at 80 days old. Breeding boars and breeding sows are immunized four times a year, and reserve pigs are immunized at 169 and 259 days old. The values of parameters $d, a_{1}$ and $a_{2}$ for individuals in different types of pig houses are shown in Table S9 in Additional file 5. The mean values of $p_{1}$ and $p_{2}$ of various pig houses and the whole farm in the stable state under different immune conditions are shown in Table S10 in Additional file 5. Based on this calculation, the individual reproduction number of pigs in various pig houses under different immune conditions is shown in Table 1. In the case of no vaccination, the basic reproduction number of individuals in all types of pig houses and whole farm is greater than 1. Under immune procedures I and II, the 

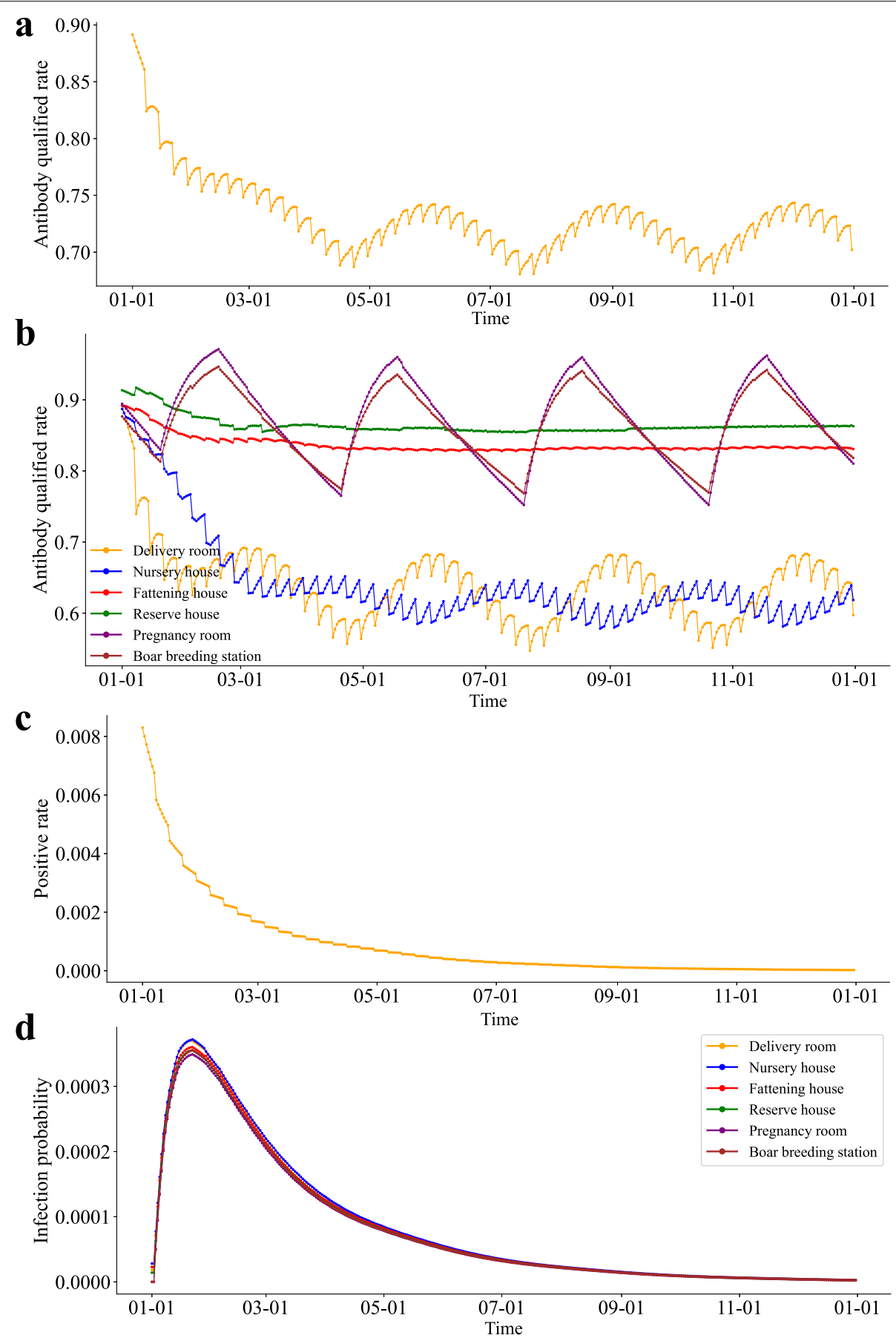

Fig. 6 When $T_{v}=\{$ January 20, April 20, July 20, October 20 $\}, T_{1}=40$ and $T_{2}=80$, the antibody qualified rate, infection situation and infection probability of the pig farm in one year. a Antibody qualified rate of the whole farm. b Antibody qualified rate in different pig houses. cThe positive rate of the whole farm. $\mathbf{d}$ The infection probability of susceptible pigs in different pig houses

individual-based control reproduction number in all types of pig houses and whole farm is less than 1 . Breeding boars are eliminated only when the boar is older than 4 years old, so the $d$ value of the boar breeding station is small. Thus, the reproduction number is the largest.

\section{Discussions}

The effect of FMD vaccines has always received a great deal of research attention. In recent decades, considerable work has been performed to study variations in the antibody protection rate under different vaccine 
Table 1 The individual reproduction number of pigs in various pig houses under different immune conditions

\begin{tabular}{llllllll}
\hline & $\begin{array}{l}\text { Delivery } \\
\text { room }\end{array}$ & $\begin{array}{l}\text { Nursery } \\
\text { house }\end{array}$ & $\begin{array}{l}\text { Fattening } \\
\text { house }\end{array}$ & $\begin{array}{l}\text { Reserve } \\
\text { house }\end{array}$ & $\begin{array}{l}\text { Pregnancy } \\
\text { room }\end{array}$ & $\begin{array}{l}\text { Boar breeding } \\
\text { station }\end{array}$ & $\begin{array}{l}\text { the whole } \\
\text { farm }\end{array}$ \\
\hline No vaccination & 1.4924 & 1.6683 & 1.9887 & 1.9647 & 2.0205 & 2.4563 & 1.7498 \\
Immune procedure I & 0.5062 & 0.5804 & 0.6344 & 0.6283 & 0.6597 & 0.7994 & 0.58 \\
Immune procedure II & 0.4329 & 0.4813 & 0.5424 & 0.5358 & 0.5590 & 0.6776 & 0.4927 \\
\hline
\end{tabular}

doses, different injection methods, different immunization procedures, different emergency immunization strategies and different populations to seek the best immunization mode to reduce the harm of the epidemic (Patil et al. 2014; Madhanmohan et al. 2012; Keeling et al. 2003; Chen et al. 2015; Xiang et al. 2015). Much of the previous work on immunization procedures was based on experimental studies on the individual animal. It has been discussed the first immunization time according to the changes in maternal antibody levels previously (Wu and Zhang 2013; Lv et al. 2011; Zhou et al. 2008; Xia et al. 2013; Yu et al. 2012). Wu and Zhang (2013) proposed that the first immunization should be performed between 30-45 days old. However, Lv and Zhou et al. proposed that the first immunization should be performed between 45 - 55 days old ( $\mathrm{Lv}$ et al. 2011; Zhou et al. 2008). Researchers studied the change law of antibodies under different immunization procedures (Ning et al. 2009; Zhang 2014). It was Ning et al. (2009) suggested that the best immunization procedure for the swine serotype O FMDV inactivated vaccine developed by LVRI included the first immunization at 50 days old and the second immunization at 90 days old. Zhang (2014) suggested that the adjusted immunization program was better than the preadjusted immunization program for bivalent inactivated oil emulsion vaccines of swine serotype $O$ and Asia 1 FMDV. The adjusted immunization procedure was as follows: the first immunization is delivered at 25 days old, the second immunization is administered at 50 days old, the third immunization is delivered at 90 days old, and then immunizations are administered once every 3 months. For breeding pigs, $\mathrm{He}$ and $\mathrm{Yu}$ (2007) demonstrated that immunization three times a year is appropriate. As noted from the above studies, since the experiment is often influenced by individual differences, different experimental methods, different types of vaccines and other factors, conclusions with relatively large differences are often reported. In this case, based on experimental data, transmission dynamics and antibody dynamics, the influence of the immunization procedure on the antibody protection level can be comprehensively studied by applying a mathematical model.

To date, there has been some work in the field of mathematics in performing research on FMD. Wada et al. (2017) applied a disease simulation model to evaluate control strategies for the 2010 Japanese outbreak of FMD and to determine the role of emergency vaccination in epidemic control. Taking cattle as an example and individuals as the research object, Mushayabasa (2020) established an ordinary differential equation dynamics model to study the effects of low-efficiency vaccines and highefficiency vaccines on the transmission dynamics of FMD. Ringa and Bauch (2014) constructed a pair approximation model of FMD to describe the spread of FMD, and their numerical simulation results showed that prophylactic vaccination was more effective than ring vaccination at the same per capita vaccination rate. The above work studies which type of vaccination method or how much immunization rate can prevent the outbreak of the epidemic and does not study the immunization procedure of a farm. In addition, the previous compartment model could not be suitable for the study of the antibody level in the body of individuals, so it could not study the problem of immunization procedure. Li et al. (Li et al. 2009) established a mathematical statistical model between antibody titer and protection rate to evaluate the efficacy of vaccines. In fact, the statistical model is a data-based model without considering antibody dynamics.

Therefore, in the current study, based on the breeding mode of large-scale farms, transmission mechanism of FMD and immunization procedure, an individual-based state probability model was established, and the relationship between inoculation time and antibody protection rate, positive rate and reproduction number was given. Based on the model, this paper considered the changes of antibody protection rate and positive rate under different first immunization time $\left(T_{1} \epsilon\{40,45,50,55,60\}\right)$, second immunization time $\left(T_{2} \in\{85,90,95,100\}\right)$ and different times of immunization ( $3-4$ times) and found the optimal immunization procedure. Specifically, commercial pigs are vaccinated at 40 days old and again at 80 days old. Reserve pigs are vaccinated at 169 and 259 days old. Breeding sows and boars are vaccinated 4 times a year.

Lin's results showed that the optimal immunization program includes the first immunization at 40 days old and the second immunization at 85 days old (Lin et al. 2019). Some differences are noted between the time of secondary immunization obtained by the method of establishing the model in this paper and that obtained by the pig experiment in the literature. This difference is attributed to the notion that the system in this paper can take into 
account the characteristics of farms inoculating vaccines in batches according to reproductive rhythm. Compared with the experimental results, the numerical simulation of the mathematical model can take into account changes in the antibody levels of all pigs in the farm over time under different conditions and save experimental costs.

In addition, different vaccines and different doses of inoculation will affect the change in antibody levels in vivo, which are related to parameters $k$ and $\delta$ in the model. Therefore, we can change the values of $k$ and $\delta$ to study the best immunization program for other vaccines or doses. In the ordinary differential model, the immune antibody loss rate $k$ is a constant and is based on the assumption that the antibody protection time obeys an exponential distribution. Compared with the actual situation, in this assumption, antibody loss is too rapid. In this model, according to the experimental data of antibody growth and decline, we fit the time series of the immune protection rate with the model provided in Additional file 4 to obtain $k(t)$ and the immunization rate $\delta(t)$, yielding more accurate results. In future studies, the gamma distribution can be used to fit $k$ and $\delta$.

In summary, the individual-based state probability model has universal applicability because it is applicable to farms of different sizes, different vaccines, different diseases and different populations. Under different backgrounds, the model parameters should have different values, and the results can be deduced by numerical simulation. This methodology not only reduces the experimental cost but also makes the results more comprehensive and propagable.

\section{Conclusions}

Based on the detection data, transmission dynamics and antibody dynamics, a probability model is established for a large-scale farm in China. The numerical simulation results reveals the following the preferred immunization procedures: commercial pigs are vaccinated at 40 days old and again at 80 days old. Reserve pigs are vaccinated at 169 and 259 days old, and breeding sows and boars are vaccinated 4 times a year. The method used in this study is applicable to different diseases, different vaccines, different doses and different populations.

\section{Methods}

\section{Model formulation}

Using pigs in the large-scale farm as nodes in the network and considering the type of pig and pig house, age, and corresponding transfer, an individual-based state probability model is established to study the effectiveness of immune programs. The time unit of the model is taken as day.
The set of pig types in the farm is denoted by $Z=$ $\{c, r, s, b\}$, where $c$ represents commercial pigs, $r$ represents reserve pigs, $s$ represents breeding sows, and $b$ represents breeding boars. We sort and number all pigs, pig houses and pens. The serial number set of all pigs in the farm is $I=\{1,2,3, \ldots, n\}$, where $n$ is the total number of pigs. The serial number set of all pig houses in the farm is $H=\{1,2,3, \ldots, m\}$, where $m$ is the total number of pig houses; the serial number set of the delivery room is $H_{1}=\left\{1,2,3, \ldots m_{1}\right\}$; the serial number set of the nursery house is $H_{2}=\left\{m_{1}+1, m_{1}+2, m_{1}+3, \ldots m_{2}\right\}$; the serial number set of the fattening house is $H_{3}=\left\{m_{2}+1, m_{2}+\right.$ $\left.2, m_{2}+3, \ldots m_{3}\right\}$; the serial number set of the reserve house is $H_{4}=\left\{m_{3}+1, m_{3}+2, m_{3}+3, \ldots m_{4}\right\}$; the serial number set of the pregnancy room is $H_{5}=\left\{m_{4}+1, m_{4}+2, m_{4}+\right.$ $\left.3, \ldots m_{5}\right\}$; and the serial number set of the boar breeding station is $H_{6}=\left\{m_{5}+1, m_{5}+2, m_{5}+3, \ldots m\right\}$. The serial number set of all pens in pig houses is $F=\{1,2,3, \ldots, g\}$, where $g$ is the total number of pens; the serial number set of pig pens in the delivery room is $F_{1}=\left\{1,2,3, \ldots, g_{1}\right\}$; the serial number set of pig pens in the nursery house is $F_{2}=$ $\left\{g_{1}+1, g_{1}+2, g_{1}+3, \ldots, g_{2}\right\}$; the serial number set of pig pens in the fattening house is $F_{3}=\left\{g_{2}+1, g_{2}+2, g_{2}+3, \ldots, g_{3}\right\}$; the serial number set of pig pens in the reserve house is $F_{4}=\left\{g_{3}+1, g_{3}+2, g_{3}+3, \ldots, g_{4}\right\}$; the serial number set of pig pens in the pregnancy room is $F_{5}=\left\{g_{4}+1, g_{4}+2, g_{4}+\right.$ $\left.3, \ldots, g_{5}\right\}$; and the serial number set of pig pens in the boar breeding station is $F_{6}=\left\{g_{5}+1, g_{5}+2, g_{5}+3, \ldots, g\right\}$. For the $i$-th $(i \in I)$ pig, namely, the $i$-th node, $z_{i}(t)$ denotes the type of the $i$-th pig at time $t$, and $z_{i}(t) \in Z ; a_{i}(t)$ denotes the days old of the $i$-th pig at time $t$, and $a_{i}(t) \geq 0 ; h_{i}(t)$ denotes the serial number of the pig house where the $i$-th pig is located at time $t$, and $h_{i}(t) \in H$; and $f_{i}(t)$ denotes the serial number of the pen where the $i$-th pig is located at time $t$, and $f_{i}(t) \in F$.

When pigs are infected with FMD by direct contact or environmental transmission, pigs need 3-4 weeks to clear the virus. During the infection period, some infected pigs are culled due to clinical symptoms. The remaining pigs can be self-healing. According to this transmission mechanism, each pig may experience four states, including susceptible, immunized, infected and recovered, which are expressed as $S, V, E$ and $R$, respectively. Therefore, for the $i$-th node, the probabilities of being in five states at time $t$ are $P_{S}^{\left(z_{i}(t), a_{i}(t), h_{i}(t), f_{i}(t)\right)}(i, t), P_{V}^{\left(z_{i}(t), a_{i}(t), h_{i}(t), f_{i}(t)\right)}(i, t)$, $P_{E}^{\left(z_{i}(t), a_{i}(t), h_{i}(t), f_{i}(t)\right)}(i, t)$, and $P_{R}^{\left(z_{i}(t), a_{i}(t), h_{i}(t), f_{i}(t)\right)}(i, t)$, respectively. In addition, the birth, transfer, and sale of pigs are reflected in the superscript $\left(z_{i}(t), a_{i}(t), h_{i}(t), f_{i}(t)\right)$. The above processes of birth, sale, transfer of pigs and state transfer due to the transmission of FMD can be simulated by Python software. At time $t$, the amount of FMDV in the environment of the $l$-th pig house is represented by $B(l, t)$, where $l \in H$. 


$$
\left\{\begin{aligned}
& a_{i}(t)=a_{i}(t-1)+1, \\
& P_{S}^{\left(z_{i}(t), a_{i}(t), h_{i}(t), f_{i}(t)\right)}(i, t)= k(t) P_{V}^{\left(z_{i}(t-1), a_{i}(t-1), h_{i}(t-1), f_{i}(t-1)\right)}(i, t-1)+\left(1-\lambda_{1}^{f_{i}(t-1)}(i, t)\right. \\
&\left.-\lambda_{2}^{h_{i}(t-1)}(i, t)-\delta(t)\right) P_{S}^{\left(z_{i}(t-1), a_{i}(t-1), h_{i}(t-1), f_{i}(t-1)\right)}(i, t-1), \\
& P_{V}^{\left(z_{i}(t), a_{i}(t), h_{i}(t), f_{i}(t)\right)}(i, t)=(1-k(t)) P_{V}^{\left(z_{i}(t-1), a_{i}(t-1), h_{i}(t-1), f_{i}(t-1)\right)}(i, t-1) \\
&+\delta(t) P_{S}^{\left(z_{i}(t-1), a_{i}(t-1), h_{i}(t-1), f_{i}(t-1)\right)}(i, t-1), \\
& P_{E}^{\left(z_{i}(t), a_{i}(t), h_{i}(t), f_{i}(t)\right)}(i, t)=(1-\eta) P_{E}^{\left(z_{i}(t-1), a_{i}(t-1), h_{i}(t-1), f_{i}(t-1)\right)}(i, t-1)+\left(\lambda_{1}^{f_{i}(t-1)}(i, t)\right. \\
&\left.+\lambda_{2}^{h_{i}(t-1)}(i, t)\right) P_{S}^{\left(z_{i}(t-1), a_{i}(t-1), h_{i}(t-1), f_{i}(t-1)\right)}(i, t-1), \\
& P_{R}^{\left(z_{i}(t), a_{i}(t), h_{i}(t), f_{i}(t)\right)}(i, t)= \mu \eta P_{E}^{\left(z_{i}(t-1), a_{i}(t-1), h_{i}(t-1), f_{i}(t-1)\right)}(i, t-1) \\
&+P_{R}^{\left(z_{i}(t-1), a_{i}(t-1), h_{i}(t-1), f_{i}(t-1)\right)}(i, t-1), \\
& B(l, t)=(1-\omega-v) B(l, t--1)+\sum_{h_{j}(t-1)=l}^{j \in I} P_{E}^{\left(z_{j}(t-1), a_{j}(t-1), h_{j}(t-1), f_{j}(t-1)\right)}(j, t-1) .
\end{aligned}\right.
$$

The variation of the above 4 probabilities with the course of infection and immunization and the state probability transfer diagram of the $i$-th pig are shown in Fig. 7, and system (1) is its corresponding system.

In this model, two modes of transmission, direct contact transmission and environmental transmission, are considered. The probabilities of the $i$-th pig being infected by the two methods at time $t$ are expressed as $\lambda_{1}^{f_{i}(t-1)}(i, t)$ and $\lambda_{2}^{h_{i}(t-1)}(i, t)$, respectively. The specific expressions for $\lambda_{1}^{f_{i}(t-1)}(i, t)$ and $\lambda_{2}^{h_{i}(t-1)}(i, t)$ and meanings of other parameters in system (1) are interpreted as follows.

(1) $\lambda_{1}^{f_{i}(t-1)}(i, t)$. Direct contact can only occur in the same pen. The probability of a susceptible pig being infected by an infected pig in the same pen through direct contact per unit time is denoted by $\beta$. Therefore, the probability that the $i$-th pig cannot be infected by the $j$-th pig in the same pen per unit time is

$$
1-\beta P_{E}^{\left(z_{j}(t-1), a_{j}(t-1), h_{j}(t-1), f_{j}(t-1)\right)}(j, t-1)
$$

The probability that the $i$-th pig is not infected by all pigs in the same pen is

$$
\prod_{\substack{j \in I, j \neq i \\ f_{j}(t-1)=f_{i}(t-1)}}\left[1-\beta P_{E}^{\left(z_{j}(t-1), a_{j}(t-1), h_{j}(t-1), f_{j}(t-1)\right)}(j, t-1)\right] .
$$

Therefore, the probability of the $i$-th pig being infected through direct contact is

$$
\begin{aligned}
\lambda_{1}^{f_{i}(t-1)}(i, t) & =1-\prod_{\substack{j \in I, j \neq i \\
f_{j}(t-1)=f_{i}(t-1)}} \\
& {\left[1-\beta P_{E}^{\left(z_{j}(t-1), a_{j}(t-1), h_{j}(t-1), f_{j}(t-1)\right)}(j, t-1)\right] . }
\end{aligned}
$$

(2) $\lambda_{2}^{h_{i}(t-1)}(i, t)$. For environmental transmission, it is necessary to consider not only the transmission caused by media or aerosols within pig houses but also the transmission caused by media and human activities among pig houses. Here, $\gamma$ is the infection rate coefficient of virus in the environment to one susceptible pig, and $p$ is the probability that pigs are exposed to viruses in the environment in different pig houses. At time $t$, the probability that the $i$-th pig is infected by the virus in the environment in the same pig house is $\gamma B\left(h_{i}(t-1), t-1\right)$; the probability that the $i$-th pig is infected by the virus in the environment in different pig houses is $\sum_{\bar{h} \neq h_{i}(t-1)} p \gamma B(\bar{h}, t-1)$. Therefore, at time $t$, the probability that the $i$-th pig is infected by FMDV in the environment is

$$
\lambda_{2}^{h_{i}(t-1)}(i, t)=\gamma B\left(h_{i}(t-1), t-1\right)+\sum_{\substack{\bar{h} \in H \\ \bar{h} \neq h_{i}(t-1)}} p \gamma B(\bar{h}, t-1) .
$$

(3) $k(t)$ : Antibody decay rate.

(4) $\delta(t)$ : Antibody growth rate.

(5) $\eta$ : Virus clearance rate.

(6) $\mu$ : The probability of self-recovery.

(7) $\alpha$ : The amount of virus excreted by the infected pig per unit time.

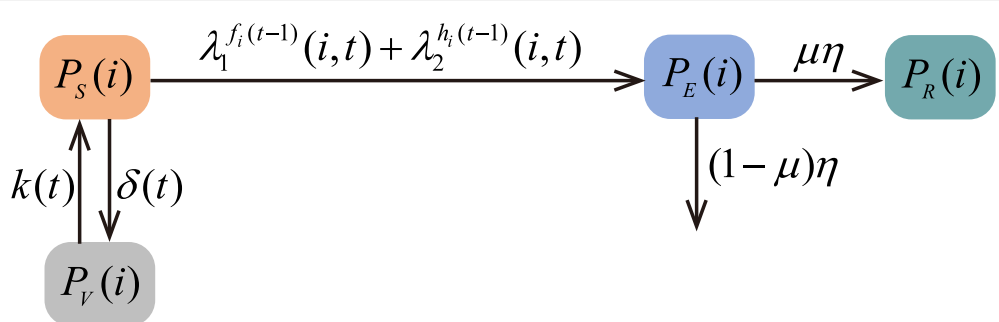

Fig. 7 State probability transfer diagram of the $i$ th pig on the farm 
(8) $\omega$ : The natural decay rate of the virus in the environment per unit time.

(9) $v$ : The disinfection rate of the virus in the environment per unit time.

If the infection process of the farm is not considered and only immunization, production and sale are considered, the system (1) becomes the following:

At time $t$, the number of pigs whose immune antibody is qualified in the whole farm is $\sum_{i \in I} P_{V}^{\left(z_{i}(t), a_{i}(t), h_{i}(t), f_{i}(t)\right)}(i, t)$, the immune antibody qualified rate in population is $\frac{\sum_{i \in I} P_{V}^{\left(z_{i}(t), a_{i}(t), h_{i}(t) f_{i}(t)\right)}(i, t)}{N(t)}$, the positive number of pigs being infected is $\sum_{i \in I} P_{E}^{\left(z_{i}(t), a_{i}(t), h_{i}(t), f_{i}(t)\right)}(i, t)$, and the positive rate in population is $\frac{\sum_{i \in I} P_{E}^{\left(z_{i}(t), a_{i}(t), h_{i}(t), f_{i}(t)\right)}(i, t)}{N(t)}$.

\section{Parameter values and initial values}

The values and sources of parameters in system (1) are interpreted as follows:

(1) Pigs infected with FMD require $3-4$ weeks to remove the virus (Alexandersen et al. 2002). So, $\eta=\frac{1}{28}$.

(2) According to the actual situation, no pigs with symptoms were culled at the farm from 2016 to 2019. So, $\mu=$ 1.

(3) The average peak amount of virus discharged by pigs per day can reach $10^{6.1} \mathrm{TCID}_{50}$ (Zhang et al. 2019). So, $\alpha=$ $10^{6.1}$.

(4) The survival time of the virus in the environment is approximately 30 days (Zhang et al. 2019). So, $\omega=\frac{1}{30}$.

(5) The farm is generally sterilized once a month. Each time disinfection rate is assumed to reach $100 \%$. Thus, $v=$ $\frac{1}{30}$.

(6) In Additional file 3, we establish an ordinary differential dynamical system (system (0.1)) for the farm to describe the spread of FMD within the pig population. By applying the data fitting of system (0.1) with real data, $\beta=0.9948$ is obtained, and its biological meaning is the product of the probability that a susceptible pig is infected by an infected pig per contact and the number of contacts in a year. The biological meaning of $\beta$ in the system (1) is the probability that a susceptible pig is infected by direct contact with an infected pig in the same pen within one day. Given that the average number of pigs per pen is 15 , the average number of direct contacts is 15 . Thus, for system (1), $\beta=0.9948 \div 365 \div 15 \approx 0.0002$.

(7) In Additional file 3, the time unit of the ordinary differential dynamic system is year, and $\gamma=4.5029 \times 10^{-10}$. The time unit of system (1) is day. Therefore, for system (1), $\gamma=4.5029 \times 10^{-10} \div 365 \approx 1.23 \times 10^{-12}$.

(8) Assume that $p=0.25$.

(9) According to Additional file 4, maternal antibodies, primary immunization and secondary immunization correspond to different $k(t)$ and $\delta(t)$. The maternal antibody of piglets reached a maximum at 7 days old. The antibody level of the first and second immunizations reached a maximum at 28 days after vaccine injection. When the immune antibody protection rate increases, $k(t)=0$. When the immune antibody protection rate decreases, $\delta(t)=0$. Commercial pigs are vaccinated at $T_{1}$ and $T_{2}$ days of age. Therefore, according to the fitting results of the antibody fluctuation rule in Additional file 4, when $z_{i}(t)=c$,

$$
k(t)= \begin{cases}0, & 1 \leq a_{i}(t) \leq 7 \\ 0.0154, & 7<a_{i}(t) \leq T_{1} \\ 0, & T_{1}<a_{i}(t) \leq T_{1}+28 \\ 0.0212, & T_{1}+28<a_{i}(t) \leq T_{2} \\ 0, & T_{2}<a_{i}(t) \leq T_{2}+28 \\ 0.0043, & a_{i}(t)>T_{2}+28\end{cases}
$$

and

$$
\delta(t)= \begin{cases}0.2299, & 1 \leq a_{i}(t) \leq 7 \\ 0, & 7<a_{i}(t) \leq T_{1} \\ 0.027, & T_{1}<a_{i}(t) \leq T_{1}+28 \\ 0, & T_{1}+28<a_{i}(t) \leq T_{2} \\ 0.0727, & T_{2}<a_{i}(t) \leq T_{2}+28 \\ 0, & a_{i}(t)>T_{2}+28\end{cases}
$$

It is assumed that the increase and decrease in the antibody protection rate in the reserve pig, breeding boar and breeding sow after vaccination is the same as that in piglets after secondary immunization. Reserve pigs are vaccinated at $T_{3}$ and $T_{4}$ days of age. Therefore, when $z_{i}(t)=r$

$$
k(t)= \begin{cases}0.0043, & a_{i}(t) \leq T_{3} \\ 0, & T_{3}<a_{i}(t) \leq T_{3}+28 \\ 0.0043, & T_{3}+28<a_{i}(t) \leq T_{4} \\ 0, & T_{4}<a_{i}(t) \leq T_{4}+28 \\ 0.0043, & a_{i}(t)>T_{4}+28\end{cases}
$$

and

$$
\delta(t)= \begin{cases}0, & a_{i}(t) \leq T_{3} \\ 0.0727, & T_{3}<a_{i}(t) \leq T_{3}+28 \\ 0, & T_{3}+28<a_{i}(t) \leq T_{4} \\ 0.0727, & T_{4}<a_{i}(t) \leq T_{4}+28 \\ 0, & a_{i}(t)>T_{4}+28\end{cases}
$$

Breeding boars and breeding sows are vaccinated thrice a year, and the set of immunization times is $T_{v}=$ January 20, May 20, September 20\}. Therefore, when $z_{i}(t) \in\{s, b\}$

$$
k(t)= \begin{cases}0, & \text { within } 28 \text { days after vaccination, } \\ 0.0043, & \text { other time, }\end{cases}
$$

and

$$
\delta(t)= \begin{cases}0.0727, & \text { within } 28 \text { days after vaccination, } \\ 0, & \text { other time. }\end{cases}
$$

In addition, according to the actual situation of the farm considered in this paper, $T_{1}=60, T_{2}=90, T_{3}=169$ and $T_{4}=259$. 


$$
\left\{\begin{aligned}
a_{i}(t)=a_{i}(t-1)+1, & \\
P_{S}^{\left(z_{i}(t), a_{i}(t), h_{i}(t), f_{i}(t)\right)}(i, t)= & k(t) P_{V}^{\left(z_{i}(t-1), a_{i}(t-1), h_{i}(t-1), f_{i}(t-1)\right)}(i, t-1) \\
& +(1-\delta(t)) P_{S}^{\left(z_{i}(t-1), a_{i}(t-1), h_{i}(t-1), f_{i}(t-1)\right)}(i, t-1), \\
P_{V}^{\left(z_{i}(t), a_{i}(t), h_{i}(t), f_{i}(t)\right)}(i, t)= & (1-k(t)) P_{V}^{\left(z_{i}(t-1), a_{i}(t-1), h_{i}(t-1), f_{i}(t-1)\right)}(i, t-1) \\
& +\delta(t) P_{S}^{\left(z_{i}(t-1), a_{i}(t-1), h_{i}(t-1), f_{i}(t-1)\right)}(i, t-1) .
\end{aligned}\right.
$$

(10) By applying system (1), the data in 2019 are used as the initial value to predict the qualified rate of immune antibodies and the level of pathogen positive rate in the pig population within one year. According to the survey in 2019, the immunization antibody qualification rate in the pig population was $89.16 \%$. The pathogen positive rate was 0.0083 ; thus, the proportion of infected pigs $(E)$ was $0.83 \%$. Assume that the number of pigs that recover $(R)$ is 0 and the number of viruses $(B)$ in the environment is 0 . According to Table S2 in Additional file 1, the total number of pigs in the farm $(N)$ is 15063 . It can be calculated that there were 1508 susceptible pigs, 13430 immune pigs and 125 infected pigs in the farm.

(11) For system (2), it is assumed that all pigs in the farm at the initial moment are susceptible. Thus, the probability of any pig being susceptible is 1 , and the probability of being immunized is 0 . We simulate the system (2) to consider the influence of different primary immunization times, secondary immunization times and immunization frequencies on the immune antibody qualified rate in the farm. The immune antibody level of breeding sows directly determines the maternal antibody level of piglets.

\section{The control reproduction number}

For one pig in a certain type of pig house, its individual basic reproduction number (Keeling and Grenfell 2000) is

$R_{0}=\int_{0}^{\infty}\left[a_{1} \beta+a_{2} \gamma h(B)+\left(N-a_{2}\right) p \gamma h(B)\right] T f(T) \mathrm{d} T$,

and its individual control reproduction number is

$R_{c}=\int_{0}^{\infty}\left[a_{1} \beta p_{1}+a_{2} \gamma h(B) p_{1}+\left(N-a_{2}\right) p \gamma h(B) p_{2}\right] T f(T) \mathrm{d} T$.

where (1) $a_{1}$ is the number of pigs in the same pen. $p_{1}$ is the proportion of susceptible pigs in the same pig house. (2) Let the right end of $\frac{d B}{d t}=\alpha E-(\omega+v) B$ in system $(0.1)$ in Additional file 3 be equal to 0 ; then, $B=\frac{\alpha E}{\omega+v}$. Therefore, at steady state, the amount of virus released into the air by a pig is $h(B)=\frac{\alpha}{\omega+v} \cdot a_{2}$ is the number of pigs in the same house. $N-a_{2}$ is the number of pigs that are not in the same pen. $p_{2}$ is the proportion of susceptible pigs in the whole farm. (3) $T$ is the infection period and obeys an exponential distribution, namely, $T \sim \operatorname{Exp}(\eta+d)$. Then, the density function is $f(T)=(\eta+d) \mathrm{e}^{-(\eta+d) T}$, and the average infection period is $\int_{0}^{\infty} T f(T) \mathrm{d} T=\frac{1}{\eta+d}$.
Therefore,

$$
\begin{aligned}
R_{c}= & p_{1}\left(\frac{1}{\eta+d}\right)\left(\beta a_{1}+\frac{\gamma \alpha a_{2}}{\omega+v}\right) \\
& +p_{2}\left(\frac{1}{\eta+d}\right)\left(\frac{p \gamma \alpha\left(N-a_{2}\right)}{\omega+v}\right),
\end{aligned}
$$

which is used as an evaluation index.

\section{Abbreviations}

FMD: Foot-and-mouth disease; FMDV: Foot-and-mouth disease virus; LVRI: Lanzhou veterinary research institute

\section{Supplementary Information}

The online version contains supplementary material available at https://doi.org/10.1186/s44149-021-00035-3.

Additional file 1: The details of the pig houses and the amount of the various pigs.

Additional file 2: The transfer figure of various types of pigs among different pig houses.

Additional file 3: Values of $\beta$ and $\gamma$.

Additional file 4: Values of $k$ and $\delta$.

Additional file 5: The values of parameters $d, a_{1}, a_{2}, p_{1}$ and $p_{2}$ for different types of pig houses.

Acknowledgements

We would like to express our sincere thanks to Yanfeng Xue and others for their guidance on model building and programming.

\section{Authors' contributions}

H.R., Z.J., X.P., M.L. and J.Z. determined the research theme and conceived and designed the research methods. Y.W. provided background materials. H.R. and J.Z. performed data analysis and programming and wrote the paper. All authors gave final approval for publication. The authors read and approved the final manuscript.

\section{Funding}

This work is supported by the National Key Research and Development Program of China (2016YFD0501501), the National Natural Science Foundation of China under Grant (11601292, 61873154, 11801398), Fund Program for the Scientific Activities of Selected Returned Overseas Professionals in Shanxi Province (20210009), General Youth Fund project in Shanxi Province (201901D211158), the 1331 Engineering Project of Shanxi Province, Scientific and Technological Innovation Programs of Higher Education Institutions in Shanxi Province (2019L0114), Key Projects of Health Commission of Shanxi Province (No. 2020XM18), and the Key Research and Development Project in Shanxi Province (202003D31011/GZ).

\section{Availability of data and materials}

All data generated or analyzed during this study are included in this published article and its supplementary information files.

\section{Declarations}




\section{Ethics approval and consent to participate} Not applicable.

\section{Consent for publication}

Not applicable.

\section{Competing interests}

The authors declare that they have no competing interests.

\section{Author details}

${ }^{1}$ Complex Systems Research Center, Shanxi University, 030006 Shanxi, China.

${ }^{2}$ Shanxi Key Laboratory of Mathematical Techniques and Big Data Analysis on Disease Control and Prevention, 030006 Shanxi, China. ${ }^{3}$ School of Mathematical Sciences, Shanxi University, 030006 Shanxi, China. ${ }^{4}$ College of Mathematics, Taiyuan University of Technology, 030024 Shanxi, China. ${ }^{5}$ The Laboratory of Animal Epidemiological Surveillance, China Animal Health \& Epidemiology Center, 266032 Shandong, China.

Received: 30 September 2021 Accepted: 23 December 2021 Published online: 18 February 2022

\section{References}

Alexandersen, S., Z. Zhang, and A. I. Donaldson. 2002. Aspects of the persistence of foot-and-mouth disease virus in animals-the carrier problem. Microbes and Infection 4(10): 1099-1110. https://doi.org/10.1016/ S1286-4579(02)01634-9.

Alexandersen, S., Z. Zhang, A. I. Donaldson, and A. Garland. 2003. The pathogenesis and diagnosis of foot-and-mouth disease. Journal of Comparative Pathology 129(1): 1-36. https://doi.org/10.1016/S00219975(03)00041-0.

Bai, X. W., P. H. Li, H. F. Bao, Z. X. Liu, and X. T. Liu. 2011. Evolution and molecular epidemiology of foot-and-mouth disease virus in China. Chinese Science Bulletin 56(21): 2191-2201. https://doi.org/10.1007/s11434-011-4563-3.

Barnett, P. V., S. J. Cox, N. Aggarwal, H. Gerber, and K. C. Mccullough. 2002. Further studies on the early protective responses of pigs following immunisation with high potency foot and mouth disease vaccine. Vaccine 20(25-26): 3197-3208. https://doi.org/10.1016/S0264-410X(02)00242-6.

Belsham, G. J., S. M. Jamal, T. Kirsten, and B. Anette. 2011. Rescue of foot-and-mouth disease viruses that are pathogenic for cattle from preserved viral rna samples. PLOS ONE 6(1): 14621. https://doi.org/10.1371/ journal.pone.0014621.

Carpenter, T. E., J. M. O'Brien, A. D. Hagerman, and B. A. McCar. 2011. Epidemic and economic impacts of delayed detection of foot-and-mouth disease: a case study of a simulated outbreak in california. Journal of Veterinary Diagnostic Investigation 23(1): 26-33. https://doi.org/10.1177/ 104063871102300104.

Chen, N. Q., F. W. Zhao, Y. Z. Mao, D. J. Zhu, H. Y. Li, and Y. Ran. 2015. Study on the growth and decline of immune antibodies of different vaccines against swine o-type foot-and-mouth disease (in chinese). Jiangxi Journal of Animal Husbandry \& Veterinary Medicine 5: 47-49. https://doi.org/CNKI: SUN:JXXS.0.2015-05-029.

Dreessche, P., and J. Watmough. 2002. Reproduction numbers and sub-threshold endemic equilibria for compartmental models of disease transmission. Mathematical Biosciences 180(1): 29-48. https://doi.org/10. 1016/s0025-5564(02)00108-6.

Gomez, S., A. Arenas, J. Borge-Holthoefer, S. Meloni, and Y. Moreno. 2010. Discrete-time markov chain approach to contact-based disease spreading in complex networks. Europhysics Letters 89(3): 38009. https://doi.org/10. 1209/0295-5075/89/38009

He, W. B., and X. L. Yu. 2007. Study on immunization program of swine with foot and mouth disease (in chinese). Hunan Journal of Animal Science \& Veterinary Medicine 6: 7-9. https://doi.org/CNKI:SUN:HNCM.0.2007-06-003.

Kardjadj, M. 2017. Foot-and-mouth disease (fmd) in the maghreb and its threat to southern European countries. Tropical Animal Health and Production 49(2): 423-425. https://doi.org/10.1007/s11250-016-1176-5.

Keeling, M. J., and B. T. Grenfell. 2000. Individual-based perspectives on r(0). Journal of Theoretical Biology 203(1): 51-61. https://doi.org/10.1006/jtbi. 1999.1064.

Keeling, M. J., M. Woolhouse, R. M. May, G. Davies, and B. T. Grenfell. 2003. Modelling vaccination strategies against foot-and-mouth disease. Nature 421(6919): 136-142. https://doi.org/10.1038/nature01343.
Li, L., A. Xing, D. Liao, M. Zhu, J. Chen, and H. Li. 2009. Fmd vaccines valuation based on virus neutralization antibody (in chinese). Chinese Journal of Preventive Veterinary Medicine 31(3): 231-235. https://doi.org/CNKI:SUN: ZGXQ.0.2009-03-019.

Lin, L. Z., C. Z. Xu, Y. Fang, H. Zhang, H. Chen, C. X. Yuan, and F. Yang. 2019. Detection of maternal antibody of pig o-type foot-and-mouth disease and evaluation of the effect of vaccine immunization (in chinese). Raising Pigs 1: 108-109. https://doi.org/10.13257/j.cnki.21-1104/s.2019.01.041.

Liu, G. M., W. Y. Lin, Q. Q. Song, K. Wang, Y. M. Liu, and Y. T. Zhang. 2019. Big data monitoring and analysis of foot-and-mouth disease vaccine immunization program (in chinese). Swine Industry Science 36(9): 74-76. https://doi.org/CNKI:SUN:TJXM.0.2019-09-023.

LV, L. X., K. W. He, Y. X. Ni, Z. H. Yu, and A. H. Mao. 2011. Growth and decline laws of maternal antibodies and immunity test against o-type foot and mouth disease in piglets at different ages in large-scale pig farms (in chinese). Jiangsu Agricultural Sciences 39(5): 304-306. https://doi.org/10. 15889/j.issn.1002-1302.2011.05.204.

Madhanmohan, M., S. B. Nagendrakumar, R. Kumar, J. Anilkumar, K. Manikumar, S. Yuvaraj, and V. A. Srinivasan. 2012. Clinical protection, sub-clinical infection and persistence following vaccination with extinction payloads of 01 manisa foot-and-mouth disease monovalent vaccine and challenge in goats and comparison with sheep. Research in Veterinary Science 93(2): 1050-1059. https://doi.org/10.1016/j.rvsc.2011.10.006.

Ministry of Agriculture. 2016. Notice of the ministry of agriculture on printing and distributing The National Foot and Mouth Disease Prevention and Control Plan (2016-2020) and The National Highly Pathogenic Avian Influenza Prevention and Control Plan (2016-2020) (in chinese). Bulletin of the Ministry of Agriculture of the People's Republic of China 9: 53-59. https://doi.org/CNKI: SUN:GNZB.0.2016-09-009.

Ministry of Agriculture. 2017. The national swine production development plan (2016-2020) (in chinese). China Agricultural Information 1: 16-22. https://doi.org/CNKI:SUN:NXTS.0.2017-01-004.

Ministry of Agriculture. 2020. Bulletin of the Ministry of Agriculture of the People's Republic of China 2: 51-54. https://doi.org/CNKI:SUN:GNZB.0. 2020-02-008.

Mushayabasa, S. 2020. Global stability and optimal control analysis of a foot-and-mouth disease model with vaccine failure and environmental transmission. Chaos, Solitons \& Fractals 132: 109568. https://doi.org/10. 1016/j.chaos.2019.109568.

Ning, H. J., Y. Zhou, B. Liu, H. Wang, and G. W. Zheng. 2009. Study on antibody fluctuation and immunizing program of changsha swine foot-and-mouth disease type o inactivated vaccine (in chinese). China Animal Health Inspection 10: 58-59. https://doi.org/CNKI:SUN:ZGDW.0.2009-10-032.

Patil, P. K., C. M. Sajjanar, C. Natarajan, and J. Bayry. 2014. Neutralizing antibody responses to foot-and-mouth disease quadrivalent (type o, a, c and asia 1) vaccines in growing calves with pre-existing maternal antibodies. Veterinary Microbiology 169(3-4): 233-235. https://doi.org/10.1016/j.vetmic. 2014.01.005

Pei, X., Z. Jin, W. Zhang, and Y. Wang. 2019. Detection of infection sources for avian influenza a(h7n9) in live poultry transport network during the fifth wave in china. IEEE Access 7: 1-1. https://doi.org/10.1109/ACCESS.2019. 2949606.

Ringa, N., and C. T. Bauch. 2014. Impacts of constrained culling and vaccination on control of foot and mouth disease in near-endemic settings: A pair approximation model. Epidemics 9: 18-30. https://doi.org/10.1016/j. epidem.2014.09.008.

Rweyemamu, M., P. Roeder, D. Mackay, K. Sumption, J. Brownlie, Y. Leforban, J.-F. Valarcher, N. J. Knowles, and V. Saraiva. 2008. Epidemiological patterns of foot-and-mouth disease worldwide. Transboundary and Emerging Diseases 55(1): 57-72. https://doi.org/10.1111/j.1865-1682.2007.01013.x.

Thompson, D. K., P. Muriel, D. Russell, P. Osborne, A. Bromley, M. Rowland, S. Creightyte, and C. Brown. 2002. Economic costs of the foot and mouth disease outbreak in the united kingdom in 2001. Revue Scientifique Et Technique 21(3): 675-687. https://doi.org/10.20506/rst.21.3.1353.

Wada, M., M. Stevenson, N. Cogger, and T. Carpenter. 2017. Evaluation of the control strategy for the 2010 foot-and-mouth disease outbreak in Japan using disease simulation. Transboundary and Emerging Diseases 64(3): 978-989. https://doi.org/10.1111/tbed.12467.

Wang, H., J. Xiao, M. Ouyang, H. Gao, J. Bei, L. Gao, X. Gao, and H. Wang. 2021. Assessment of foot-and-mouth disease risk areas in mainland China based spatial multi-criteria decision analysis. https://doi.org/10.21203/rs.3.rs424752/v1. 
Wernery, U., and J. Kinne. 2012. Foot and mouth disease and similar virus infections in camelids: a review. Revue Scientifique Et Technique 31(3): 907-918. https://doi.org/10.1016/j.jvc.2012.07.004.

Wu, Z. Y., and J. Zhang. 2013. Growth and decline laws of maternal antibodies of swine o-type foot and mouth disease and its interference effect on vaccine immunity (in chinese). Journal of Anhui Agricultural Sciences 41 (14): 6292-6293. https://doi.org/10.13989/j.cnki.0517-6611.2013.14.165.

Xia, C. F., F. Z. Jin, and X. J. Ren. 2013. Exploration on the first immunization time of pig foot-and-mouth disease (in chinese). Chinese Abstracts of Animal Husbandry and Veterinary Medicine 29(6): 91. https://doi.org/CNKl: SUN:ZXWA.0.2013-06-087.

Xiang, C., R. Wang, J. Zhao, L. Wei, Y. Wei, and Y. Hui. 2015. Dynamics anaiysis of immune antibodity of type o of porcine foot-and-mouth disease vaccine (in chinese). Journal of Anhui Agricultural Sciences 43(8): 109-111. https:// doi.org/10.13989/j.cnki.0517-6611.2015.08.043.

Yu, Q., J. Li, Z. Zhou, C. Li, Q. He, L. Ye, N. Du, and Y. Meng. 2012. Changes of maternal antibody level of o-type foot-and-mouth disease and test on immunization effect in pig (in chinese). Journal of Xichang College (Natural Science Edition) 26(2): 22-24. https://doi.org/CNKI:SUN:XCNY.0.2012-02009.

Zhang, J., Z. Jin, and Y. Yuan. 2019. Assessing the spread of foot and mouth disease in mainland China by dynamical switching model. Journal of Theoretical Biology 460: 209-219. https://doi.org/10.1016/j.jtbi.2018.09.027.

Zhang, X. 2014. Study on the adjustment of fmd's immunization program and its immune effect (in chinese). Sichuan Animal \& Veterinary Sciences 41 (9): 29-31. https://doi.org/CNKI:SUN:SCXS.0.2014-09-023.

Zhou, Y., H. Wang, L. P. Wang, H. J. Ning, and K. X. Yan. 2008. Test on maternal antibody dynamics against foot and mouth disease type $\mathrm{o}$ in piglets (in chinese). Journal of Anhui Agricultural Sciences 7: 2770-2771. https://doi. org/10.13989/j.cnki.0517-6611.2008.07.111.

\section{Publisher's Note}

Springer Nature remains neutral with regard to jurisdictional claims in published maps and institutional affiliations.

Ready to submit your research? Choose BMC and benefit from:

- fast, convenient online submission

- thorough peer review by experienced researchers in your field

- rapid publication on acceptance

- support for research data, including large and complex data types

- gold Open Access which fosters wider collaboration and increased citations

- maximum visibility for your research: over $100 \mathrm{M}$ website views per year

At $\mathrm{BMC}$, research is always in progress.

Learn more biomedcentral.com/submissions 